\title{
Label-free photoacoustic microscopy of cytochromes
}

Chi Zhang

Yu Shrike Zhang

Da-Kang Yao

Younan Xia

Lihong V. Wang

\section{O SPIE}




\section{Label-free photoacoustic microscopy of cytochromes}

\author{
Chi Zhang, ${ }^{a}$ Yu Shrike Zhang, ${ }^{b}$ Da-Kang Yao, ${ }^{a}$ \\ Younan Xia, ${ }^{b}$ and Lihong V. Wang ${ }^{a}$ \\ a Washington University in St. Louis, Department of Biomedical \\ Engineering, St. Louis, Missouri 63130 \\ ${ }^{b}$ Georgia Institute of Technology and Emory University, The Wallace $\mathrm{H}$. \\ Coulter Department of Biomedical Engineering, Atlanta, Georgia 30332
}

\begin{abstract}
Photoacoustic microscopy (PAM) has achieved submicron lateral resolution in showing subcellular structures; however, relatively few endogenous subcellular contrasts have so far been imaged. Given that the hemeprotein, mostly cytochromes in general cells, is optically absorbing around the Soret peak ( 420 nm), we implemented labelfree PAM of cytochromes in cytoplasm for the first time. By measuring the photoacoustic spectra of the oxidized and reduced states of fibroblast lysate and fitting the difference spectrum with three types of cytochromes, we found that the three cytochromes account for more than half the optical absorption in the cell lysate at $420 \mathrm{~nm}$ wavelength. Fixed fibroblasts on slides were imaged by PAM at 422 and $250 \mathrm{~nm}$ wavelengths to reveal cytoplasms and nuclei, respectively, as confirmed by standard staining histology. PAM was also applied to label-free histology of mouse ear sections by showing cytoplasms and nuclei of various cells. PAM of cytochromes in cytoplasm is expected to be a high-throughput, label-free technique for studying live cell functions, which cannot be accomplished by conventional histology. ( $) 2013$ Society of Photo-Optical Instrumentation Engineers (SPIE) [DOI: 10.1117/1.JBO.18.2.020504]
\end{abstract}

Keywords: photoacoustic microscopy; photoacoustic spectroscopy; cytochrome; mitochondria; label-free.

Paper 12680LRR received Oct. 16, 2012; revised manuscript received Jan. 15, 2013; accepted for publication Jan. 16, 2013; published online Jan. 31, 2013.

Photoacoustic microscopy (PAM) has the unique advantages of imaging optical absorption with $100 \%$ sensitivity and imaging the functional parameters associated with endogenous lightabsorbing biomolecules. Historically, label-free PAM has been successfully applied to in vivo imaging of hemoglobin and melanin, two major sources of endogenous optical absorption in biological tissue in the visible spectral range. Recently, additional photoacoustic contrasts have been demonstrated by exciting myoglobin ${ }^{1}$ and bilirubin ${ }^{2}$ with visible illumination, DNA and RNA in nuclei ${ }^{3}$ with ultraviolet (UV) illumination, and water $^{4}$ and lipid ${ }^{5}$ with near-infrared illumination. In fact, PAM can potentially image any molecule that has sufficient absorption at specific wavelengths.

Optical-resolution PAM has achieved submicron lateral resolution in showing subcellular structures $;{ }^{6}$ however, few endogenous subcellular contrasts apart from DNA and RNA in cell

Address all correspondence to: Lihong V. Wang, Washington University in St. Louis, Department of Biomedical Engineering, St. Louis, Missouri 63130. Tel: +(314) 935-6152; Fax: +(314) 935-7448; E-mail: Ihwang@wustl.edu nuclei $^{3}$ have so far been imaged by PAM. Here, we hypothesize that hemeprotein in cytoplasm can be imaged by PAM around the Soret peak $(\sim 420 \mathrm{~nm})$. Hemoglobin and myoglobin, two types of hemeprotein, exist mainly in red blood cells and muscle cells, respectively. In other cells, the most common hemeproteins are cytochromes, mainly located in mitochondria, whose main function is electron transport using the heme group. Previous spectrophotometric results have provided evidence that cytochromes are a major source of endogenous subcellular optical absorption at their absorption peaks. ${ }^{7}$ Photothermal technologies have been utilized to image mitochondria in cells, where the absorption source has sometimes been assumed to be mainly cytochrome $c,{ }^{8}$ but the assumption has not been verified. ${ }^{9}$ In this study, we analyzed the absorption origins in cells by photoacoustic spectroscopy.

Label-free PAM of cytochromes in cytoplasm is expected to be a useful technique for studying live cell functions, such as how the release of cytochrome $c$ from mitochondria regulates apoptosis. Label-free PAM avoids one concern about fluorescence microscopy that the fluorescent labeling probes may disturb the function of biomolecules and may have an insufficient density. For example, Mitotracker® labeling has been found to affect mitochondrial permeability and respiration. ${ }^{10}$ Moreover, by imaging cytoplasm and nuclei without labeling, PAM can provide higher throughput than standard hematoxylin and eosin (H\&E) histology, and even image live tissues in 3D in situ without sectioning. In this paper, by using optical illumination around the Soret peak, we applied PAM to image cytochromes in the cytoplasm of fixed cells and of histological sections.

We built a free-space PAM system for cytochrome imaging, shown in Fig. 1. An integrated diode-pumped Q-switched laser and optical parametric oscillator system (NT242-SH, Ekspla) generated laser pulses ( $5 \mathrm{~ns}$ pulse width, $1 \mathrm{KHz}$ repetition rate) with a tunable wavelength range from 210 to $2600 \mathrm{~nm}$. The laser pulses were sequentially filtered by an iris $(2 \mathrm{~mm}$ aperture), focused by a condenser lens, filtered by a $50 \mu \mathrm{m}$ pinhole, and focused again by an objective. The objective for visible light (46 07 15, Zeiss) has a 0.60 numerical aperture (NA), and the one for UV light (LMU-20X-UVB, Thorlabs) has a 0.40 NA. The focused laser pulse generated a spatially and temporally abrupt temperature rise in the focal zone inside the sample and thereby excited photoacoustic waves. The photoacoustic waves were detected by a focused ultrasonic transducer (customized with $40 \mathrm{MHz}$ central frequency, $80 \%$ bandwidth, and $0.50 \mathrm{NA}$ ) coupled by water, and then amplified, digitized at $1 \mathrm{GS} / \mathrm{s}$ (PCI-5152, National Instruments), and finally recorded by a computer. The relative optical absorption at the focal point was calculated by the amplitude of the photoacoustic signals. Three-dimensional (3D) imaging was realized by two-dimensional (2D) raster scanning of the sample while converting the arrival time of each photoacoustic signal to depth. Here, since the sample thickness used in this paper was comparable to or even smaller than the axial resolution ( $\sim 40 \mu \mathrm{m}$, as determined by the acoustic bandwidth), we show only 2D maximum-amplitude projection images projected along the depth direction.

We used photoacoustic spectroscopy to analyze the origination of absorption around the Soret peak in fibroblasts (NIH/ 3T3, ATCC), the most common cells in the connective tissues

0091-3286/2013/\$25.00 (C) 2013 SPIE 


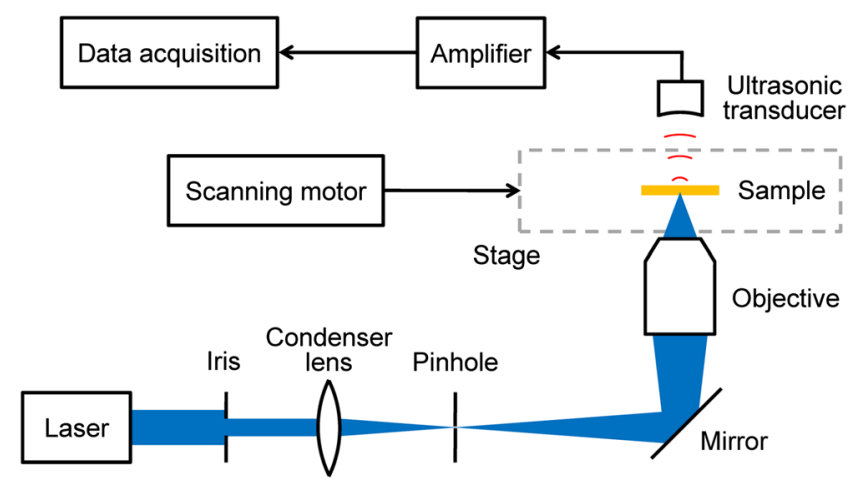

Fig. 1 Schematic of the PAM system.

of animals. Because both hemeproteins and other chromophores in cytoplasm absorb light, we analyzed the difference absorption spectrum between the oxidized and reduced states of cells, where the contributions from the chromophores other than hemeproteins were mostly removed. Most other chromophores do not have oxidized and reduced states like hemeproteins and should have approximately the same spectrum in the two states of cells. Here, the hemeproteins consist of mainly cytochromes, including cytochrome $c$, cytochrome $b$, and cytochrome $a, a_{3}$ (cytochrome $c$ oxidase), whose difference molar extinction spectra are shown in Fig. 2(a) (data from Refs. 11 and 12). We measured the average photoacoustic signal amplitude (normalized by the laser pulse energy) from air-oxidized and sodium-dithionite-reduced cell lysates,${ }^{13}$ respectively, at multiple wavelengths around the Soret peak. The difference photoacoustic spectrum is shown in Fig. 2(b). The photoacoustic spectrum was fitted with the spectrum of a mixture of $21 \pm 12 \%$ (molar ratio, mean \pm standard error) cytochrome $c$,
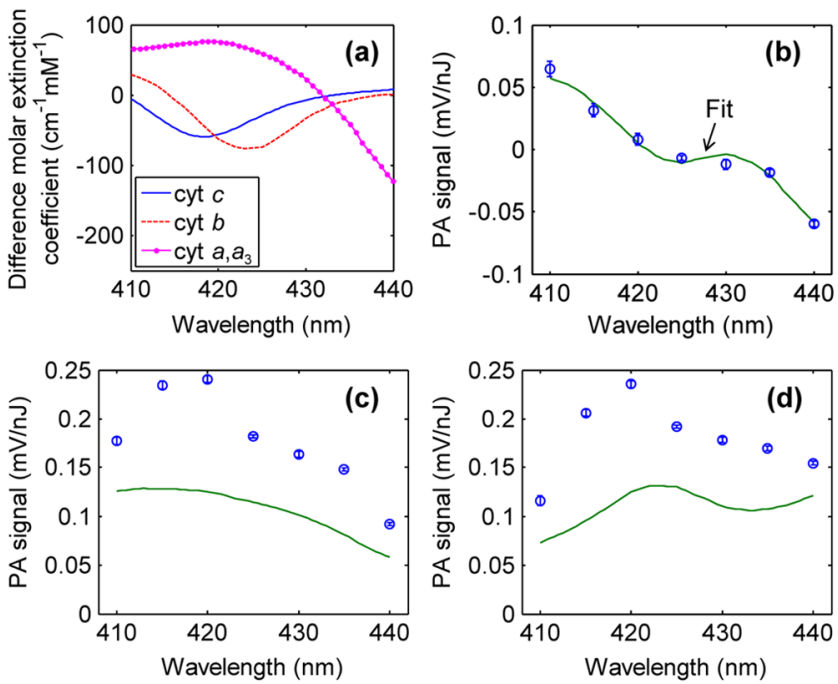

Fig. 2 Absorption spectra. (a) Difference (oxidized-reduced) molar extinction spectra of cytochrome $c$, cytochrome $b$, and cytochrome $a, a_{3}$. (b) Difference photoacoustic (PA) spectrum of the fibroblast sample between the oxidized and reduced states. The PAM measurement (circles) is fitted with the spectrum of a mixture of $21 \%$ cytochrome $C$, $43 \%$ cytochrome $b$, and $36 \%$ cytochrome $a, a_{3}$ (line). (c) PA spectrum of the oxidized fibroblast sample (circles). (d) PA spectrum of the reduced fibroblast sample (circles). The PAM measurements (circles) in (c) and (d) are compared with the spectra of the mixture (lines) in the oxidized and reduced states, respectively, according to the component concentrations calculated from (b).
$43 \pm 9 \%$ cytochrome $b$, and $36 \pm 4 \%$ cytochrome $a, a_{3}$. The coefficient of determination $\left(R^{2}\right)$ was 0.98 . The accuracy of this result, however, is subject to the possible presence of other neglected hemeproteins (such as cytochrome $\mathrm{p} 450$, nitric oxide synthases, and myeloperoxidase) with similar spectra. Then the photoacoustic spectra of the oxidized and reduced cell lysates were compared with the spectra of the calculated cytochrome mixture in the oxidized and reduced states, respectively, as shown in Fig. 2(c) and 2(d). It can be seen that the mixture of three types of cytochromes accounts for more than half of the optical absorption for the oxidized and reduced fibroblasts, respectively, at $420 \mathrm{~nm}$ wavelength (the absorption peak). This calculation of the contribution of the three cytochrome species may be an underestimation as it is based on the assumption that the three cytochromes are fully oxidized in air (any cytochrome partially reduced in the initial aerobic state would not contribute to the calculation of the total cytochrome content by our method). However, it is also possible that the remaining absorption in the fibroblasts originates from other sources, such as flavoproteins, nicotinamide adenine dinucleotide, or other neglected hemeproteins.

Next, we implemented label-free PAM of fixed fibroblasts. The fibroblasts were first maintained in Dulbecco's Modified Eagle $₫$ medium supplemented with $10 \%$ fetal bovine serum and $1 \%$ penicillin-streptomycin. Then the cells were seeded onto quartz cover glasses (sterilized in $70 \%$ ethanol for $2 \mathrm{~h}$ and washed with phosphate-buffered saline) at a density of $2 \times 10^{4}$ cells $/ \mathrm{cm}^{2}$ and allowed to attach and spread overnight. At last the cells were fixed in $3.7 \%$ formaldehyde for $30 \mathrm{~min}$ and gently washed with water. The fibroblast cytoplasms were imaged by PAM at $422 \mathrm{~nm}$ wavelength (where the laser provides stronger and more stable pulse energy than at $420 \mathrm{~nm}$ ) and $200 \mathrm{~nJ}$ pulse energy, as shown in green in Fig. 3(a). The fibroblast nuclei were also imaged by PAM at $250 \mathrm{~nm}$ wavelength, as shown in blue in Fig. 3(b). Figure 3(c) is a superimposed image of Fig. 3(a) and 3(b). While most cytochromes are located in mitochondria, some cytochromes and other subcellular chromophores have also been identified in extramitochondrial locations. The low contrast between the mitochondria and the other parts of the cytoplasm, along with the insufficient axial resolution of PAM, caused the entire cytoplasm to be shown without individual mitochondria being resolved. As a comparison, the cells were then stained with MitoTracker® Green FM (Invitrogen) for mitochondria and 4', 6-diamidino-2-phenylindole (Invitrogen) for nuclei. The stained cells were imaged by bright-field fluorescence microscopy. The fluorescence image shown in Fig. 3(d) matches well with the PAM image shown in Fig. 3(c). Here the mitochondria were not resolved by fluorescence microscopy either because the bright-field microscopy has insufficient axial resolution. However, individual mitochondria of many of these fibroblasts were resolved by confocal optical microscopy (not shown here) due to its finer axial resolution.

We then imaged a histological frozen section of a mouse ear by label-free PAM. The freshly excised mouse ear was frozen rapidly to $-20^{\circ} \mathrm{C}$, sectioned in parallel to the skin surface at $5 \mu \mathrm{m}$ thickness, and fixed with acetone for $15 \mathrm{~min}$. A dualwavelength PAM image of the mouse ear section is shown in Fig. 4(a). The cytoplasms (imaged at $422 \mathrm{~nm}$ wavelength) are shown in pink, and the nuclei (imaged at $250 \mathrm{~nm}$ wavelength) are shown in blue. Myocytes and adipocytes can be clearly identified in the image. An optical microscopy image of the 
(a)

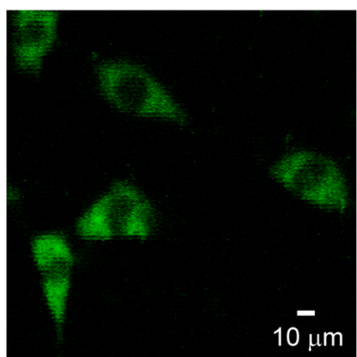

Optical absorption at $422 \mathrm{~nm}$ 0

(c)

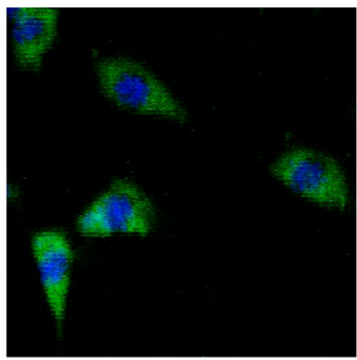

(b)

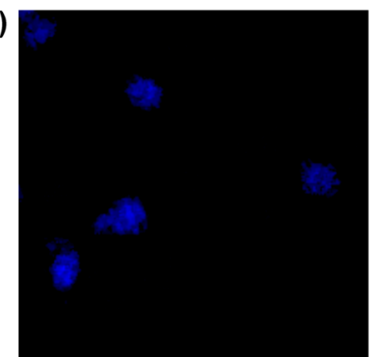

Optical absorption at $250 \mathrm{~nm}$ 0

(d)

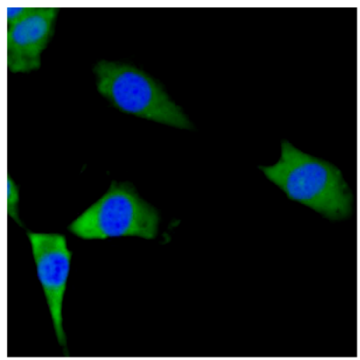

Fig. 3 PAM and fluorescence microscopy of fibroblasts. (a) Label-free PAM image of fixed but unstained fibroblasts acquired at $422 \mathrm{~nm}$ wavelength. (b) Label-free PAM image acquired at $250 \mathrm{~nm}$ wavelength. (c) Superimposed image of (a) and (b). (d) Fluorescence microscopy image of the cells with mitochondria stained in green and nuclei stained in blue.

(a)

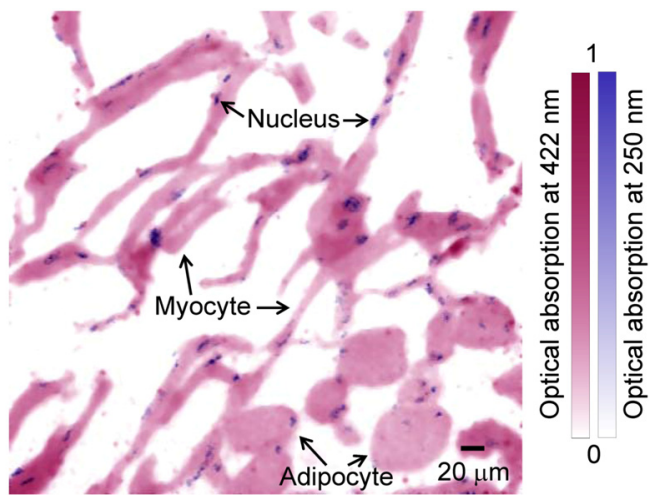

(b)

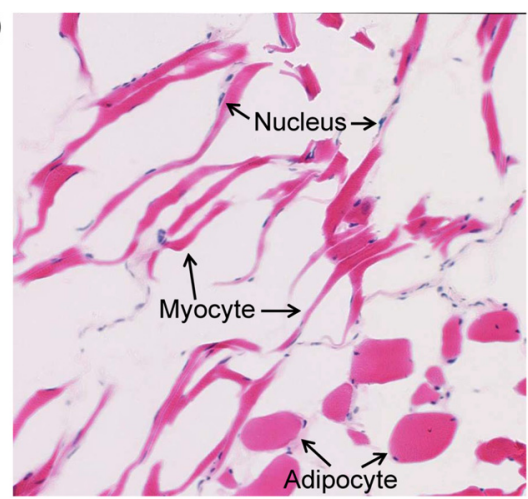

Fig. 4 Imaging of a mouse ear section. (a) Dual-wavelength label-free PAM image. Absorption at $422 \mathrm{~nm}$ is shown in pink, and absorption at $250 \mathrm{~nm}$ is shown in blue. (b) Optical microscopy image acquired after hematoxylin and eosin staining. same mouse ear section with H\&E staining [Fig. 4(b)] matches well with the unstained PAM image. Therefore, PAM has the potential for label-free high-throughput histology by imaging specific substances of interest (e.g., cytochromes, DNA, RNA, lipid, hemoglobin, and melanin) at selected wavelengths.

In summary, we have realized label-free PAM of cytochromes in cytoplasm, along with other subcellular chromophores. The specificity of PAM to mitochondria can be further studied by imaging cells with specific subcellular mitochondrial locations and by comparing the contrast of concentrated mitochondria with that of residual cytoplasm. We also expect to further develop label-free PAM for real-time functional imaging of live cells. With the technical developments, highresolution functional imaging of live cells can be realized by multiwavelength PAM, such as imaging the ratio of reduced to oxidized cytochromes, which is difficult for fluorescence microscopy.

\section{Acknowledgments}

This work was sponsored in part by National Institutes of Health (NIH) grants R01 EB000712, R01 EB008085, R01 CA134539, U54 CA136398, R01 CA157277, and R01 CA159959. L.W. has a financial interest in Microphotoacoustics Inc. and Endra Inc., which did not support this work.

\section{References}

1. C. Zhang et al., "Label-free photoacoustic microscopy of myocardial sheet architecture," J. Biomed. Opt. 17(6), 060506 (2012).

2. Y. Zhou et al., "Photoacoustic microscopy of bilirubin in tissue phantoms," J. Biomed. Opt. 17(12), 126019 (2012).

3. D.-K. Yao et al., "In vivo label-free photoacoustic microscopy of cell nuclei by excitation of DNA and RNA," Opt. Lett. 35(24), 4139-4141 (2010).

4. Z. Xu, Q. Zhu, and L. V. Wang, "In vivo photoacoustic tomography of mouse cerebral edema induced by cold injury," J. Biomed. Opt. 16(6), 066020 (2011).

5. H.-W. Wang et al., "Label-free bond-selective imaging by listening to vibrationally excited molecules," Phy. Rev. Lett. 106(23), 238106 (2011).

6. C. Zhang, K. Maslov, and L. V. Wang, "Subwavelength-resolution label-free photoacoustic microscopy of optical absorption in vivo," Opt. Lett. 35(19), 3195-3197 (2010).

7. B. Thorell and B. Chance, "Microspectrography of respiratory enzymes within the single, mammalian cell under different metabolic conditions," Exp. Cell Res. 20(1), 43-55 (1960).

8. A. V. Brusnichkin et al., "Ultrasensitive label-free photothermal imaging, spectral identification, and quantification of cytochrome $c$ in mitochondria, live cells, and solutions," J. Biophotonics 3(12), 791-806 (2010).

9. D. Lasne et al., "Label-free optical imaging of mitochondria in live cells," Opt. Exp. 15(21), 14184-14193 (2007).

10. L. Scorrano et al., "Chloromethyltetramethylrosamine (Mitotracker Orange $\mathrm{e}^{\mathrm{TM}}$ ) induces the mitochondrial permeability transition and inhibits respiratory complex I. Implications for the mechanism of cytochrome c release," J. Biol. Chem. 274(35), 24657-24663 (1999).

11. G.-L. Laio and G. Palmer, "The reduced minus oxidized difference spectra of cytochromes $a$ and $a_{3}$," Biochim. Biophys. Acta 1274(3), 109-111 (1996).

12. Biomedical Optics Research Laboratory (BORL), "Cytochrome spectra," Department of Medical Physics and Bioengineering, University College London, http://www.medphys.ucl.ac.uk/research/borl/research/NIR_ topics/spectra/spectra.htm (18 October 2005).

13. R. A. Capaldi, M. F. Marusich, and J.-W. Taanman, "Mammalian cytochrome-c oxidase: characterization of enzyme and immunological detection of subunits in tissue extracts and whole cells," Method. Enzymol. 260, 117-132 (1995). 